\title{
Biodiversité des plantations d'eucalyptus
}

\section{Jacques TASSIN ${ }^{1}$}

Aimé P. MisSAMBA-LOLA ${ }^{2}$

Jean-Noël MARIEN ${ }^{1,2}$

\author{
${ }^{1}$ Cirad \\ Upr Bsef \\ Biens et services des écosystèmes \\ forestiers tropicaux \\ Campus international de Baillarguet \\ 34398 Montpellier Cedex 05 \\ France \\ 2 Ur2pi \\ BP 1291 \\ Pointe-Noire \\ Congo
}

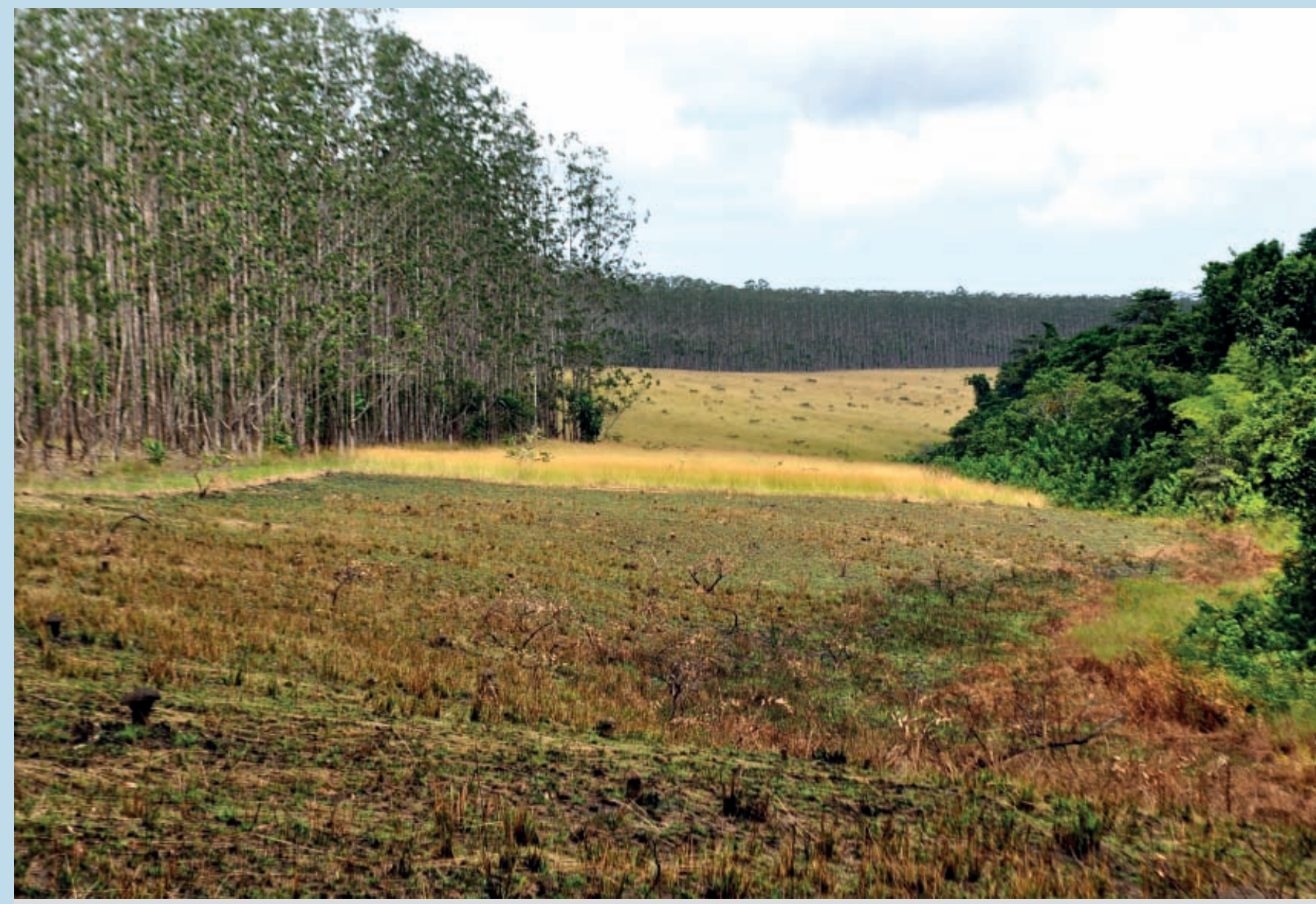

Les plantations réalisées sur des savanes herbeuses régulièrement parcourues par les feux concourent généralement à accroître la biodiversité, Pointe-Noire au Congo.

Photo J. Tassin. 


\section{RÉSUMÉ}

\section{BIODIVERSITÉ DES PLANTATIONS D'EUCALYPTUS}

Les plantations d'eucalyptus souffrent d'une image négative relative à leur diversité biologique. Une représentation univoque paraît néanmoins abusive dans la mesure où l'on s'adresse alors à des modèles de référence, des échelles, des contextes et des objectifs qui peuvent être très divers. Il convient de clarifier les processus agissant, à un niveau d'organisation du vivant qui leur est souvent spécifique, sur la biodiversité des plantations d'eucalyptus. Trois niveaux principaux sont distingués : la parcelle de plantation, le paysage environnant, la région. La diversité génétique des eucalyptus plantés, leur allélopathie potentielle, l'éclairement et la production de ressources à l'égard de la faune peuvent s'envisager à l'échelle de la parcelle plantée. Une approche paysagère permet alors, à un niveau supérieur, d'appréhender les flux d'espèces, d'éventuelles formes de conversion et des effets de protection directs ou indirects. Le niveau régional, enfin, relève de l'investigation des facteurs climatiques, mais aussi anthropiques, qui pèsent à leur tour sur l'expression de la biodiversité des plantations d'eucalyptus. D'après l'approche hiérarchique, il paraît avantageux que l'ensemble des critères, indicateurs et vérificateurs identifiés pour une sylviculture durable des plantations d'eucalyptus soient également appliqués selon de tels niveaux d'organisation. Mais en amont d'une telle démarche, il importe d'expliciter l'ensemble des attentes sociales dévolues aux plantations d'eucalyptus, de manière à orienter convenablement les techniques retenues, les pratiques sylvicoles mises en œuvre, et les modes de gestion de tels peuplements.

Mots-clés : diversité biologique, approche hiérarchique, évaluation de la biodiversité, certification, pratiques sylvicoles, impact environnemental.

\section{ABSTRACT}

\section{BIODIVERSITY OF EUCALYPTUS PLANTATIONS}

Eucalyptus plantations have a negative image in terms of their biological diversity. It would, however, be unfair to oversimplify this representation, since it brings into play a broad diversity of reference models, scales, contexts and objectives. It is appropriate to clarify the processes affecting the biodiversity of eucalyptus plantations according to specific levels of organization. Three main levels can be identified: the plantation plot itself, the surrounding landscape and the region. The genetic diversity of planted eucalyptus trees, their potential allelopathic relations with other organisms, the light conditions in the plantation and the production of wildlife resources can be examined on the scale of the plantation plot. On a higher level, the landscape approach can cast light on the flow of species, possible forms of conversion and the direct or indirect protection effects. Finally, the regional approach involves the investigation of climatic, but also anthropogenic factors which, in turn, exert pressure on the biodiversity of eucalyptus plantations. According to the hierarchy approach, it would seem beneficial to apply all of the criteria, indicators and means of verification identified for the sustainable silviculture of eucalyptus plantations equally in accordance with such levels of organisation. But prior to taking such a step, it is important to clarify the range of social expectations focused on eucalyptus plantations in order to apply the techniques selected, the silvicultural practices applied and the type of management of such stands in an acceptable manner.

Keywords: biological diversity, hierarchy approach, biodiversity evaluation, certification, silvicultural practices, environmental impact.

\section{RESUMEN}

\section{BIODIVERSIDAD EN PLANTACIONES DE EUCALIPTOS}

Las plantaciones de eucaliptos sufren de una imagen negativa en cuanto a su diversidad biológica. No obstante, una representación unívoca parece excesiva en la medida en que se destina a modelos de referencia, escalas, contextos y objetivos que pueden ser muy diversos. Conviene esclarecer los procesos que actúan, en un nivel de organización de los organismos vivos que suele ser específico, sobre la biodiversidad de las plantaciones de eucaliptos. Se diferencian tres niveles principales: la parcela de plantación, el paisaje circundante y la región. La diversidad genética de los eucaliptos plantados, su potencial alelopático, la iluminación y la producción de recursos respecto a la fauna pueden considerarse a escala de la parcela plantada. Un enfoque paisajístico permite entonces, a un nivel superior, entender los flujos de especies, las posibles formas de conversión y los efectos de protección directos o indirectos. El nivel regional, por último, consiste en la investigación de los factores climáticos, y también antrópicos, que a su vez se ciernen sobre la expresión de la biodiversidad de las plantaciones de eucaliptos. Según el enfoque jerárquico, parece más útil que el conjunto de criterios, indicadores y verificadores identificados para una silvicultura sostenible de las plantaciones de eucalipto se aplique también siguiendo estos niveles de organización. Pero previamente a este planteamiento, es importante explicitar el conjunto de expectativas sociales atribuidas a las plantaciones de eucalipto para, de este modo, orientar adecuadamente las técnicas elegidas, las prácticas silvícolas aplicadas y los modos de manejo de estas masas.

Palabras clave: diversidad biológica, enfoque jerárquico, evaluación de la biodiversidad, certificación, prácticas silvícolas, impacto ambiental. 


\section{Introduction}

Les plantations d'eucalyptus couvrent de 13 à 19 millions d'hectares distribués dans plus de 70 pays (FAO, 1995). Elles recouvrent une multitude de réalités, selon qu'elle sont par exemple dans un système à dominante industrielle ou paysanne. Le succès du genre Eucalyptus résulte d'une heureuse conciliation entre rapidité de croissance, rusticité, plasticité écologique, aptitude au recépage, productivité et fourniture de produits industriels ou domestiques très diversifiés. Avec les pins, les eucalyptus recouvrent $30 \%$ des plantations actuelles (FAO, 2005).

Or, ces plantations revêtent également une dimension culturelle exacerbant parfois des craintes latentes. L'intrusion omniprésente de cet arbre dans les paysages anthropogènes tropicaux et méditerranéens vient bousculer une représentation idéale de la sylviculture où s'harmonisent la valorisation de l'espace, l'esthétique paysagère, le foncier, l'emploi et la filière bois. C'est cependant précisément sur le terrain de la biodiversité que s'est aujourd'hui cristallisée la polémique sur les conséquences socio-environnementales de la sylviculture des plantations d'eucalyptus.

Selon la définition de la Convention sur la diversité biologique, la biodiversité est la variabilité observée en un lieu au sein des espèces, entre espèces, et entre les écosystèmes en présence (NATIONS UNIES, 1992). L'importance du maintien de la biodiversité au sein des écosystèmes forestiers a connu un regain de vigueur dans les quinze dernières années avec l'émergence de la gestion forestière durable, soutenue au plan commercial par des processus de certification tels que le Forest Stewardship Council (Fsc) et le Programme for the Endorsement of Forest Certification (Pefc).

L'assimilation métaphorique des plantations d'eucalyptus à des déserts biologiques a précédé, puis accompagné cette évolution (POORE, FrIES, 1985 ; ZOBEL et al., 1987 ; SUNDER, 1995). Si l'écologie ne peut apaiser une controverse débordant aussi largement son champ, du moins s'agit-il d'expliciter les effets de la sylviculture d'eucalyptus sur la biodiversité. Il s'agit de hiérarchiser les facteurs déterminant la biodiversité des plantations d'eucalyptus, de manière à édicter des règles de gestion favorables (BRosset, 1997 ; MARIen, 2000 ; Geldenhuys, 1997). Ces facteurs recouvrent principalement les conditions climatiques et édaphiques en présence, les processus liés au mouvement des espèces dans l'espace, de même que leur évolution dans le temps ; mais aussi l'ensemble des perturbations naturelles ou induites par l'homme (CARNus et al., 2006). La diversité d'échelles spatiales et temporelles en jeu entretient en effet une certaine confusion lorsque ces dernières ne sont pas précisément explicitées.

Aussi se justifie-t-il de recourir à une approche hiérarchique (Allen, StARr, 1982 ; Noss, 1990), comme proposé dans cet article. La sylviculture d'eucalyptus peut dès lors être envisagée selon trois principaux niveaux d'organisation emboîtés : la parcelle, le paysage environnant (au sens écologique du terme), la région. Mais puisque la biodiversité revêt aujourd'hui une dimension normative, il s'agit dans un premier temps de s'interroger sur les modèles auxquels il convient de se référer.

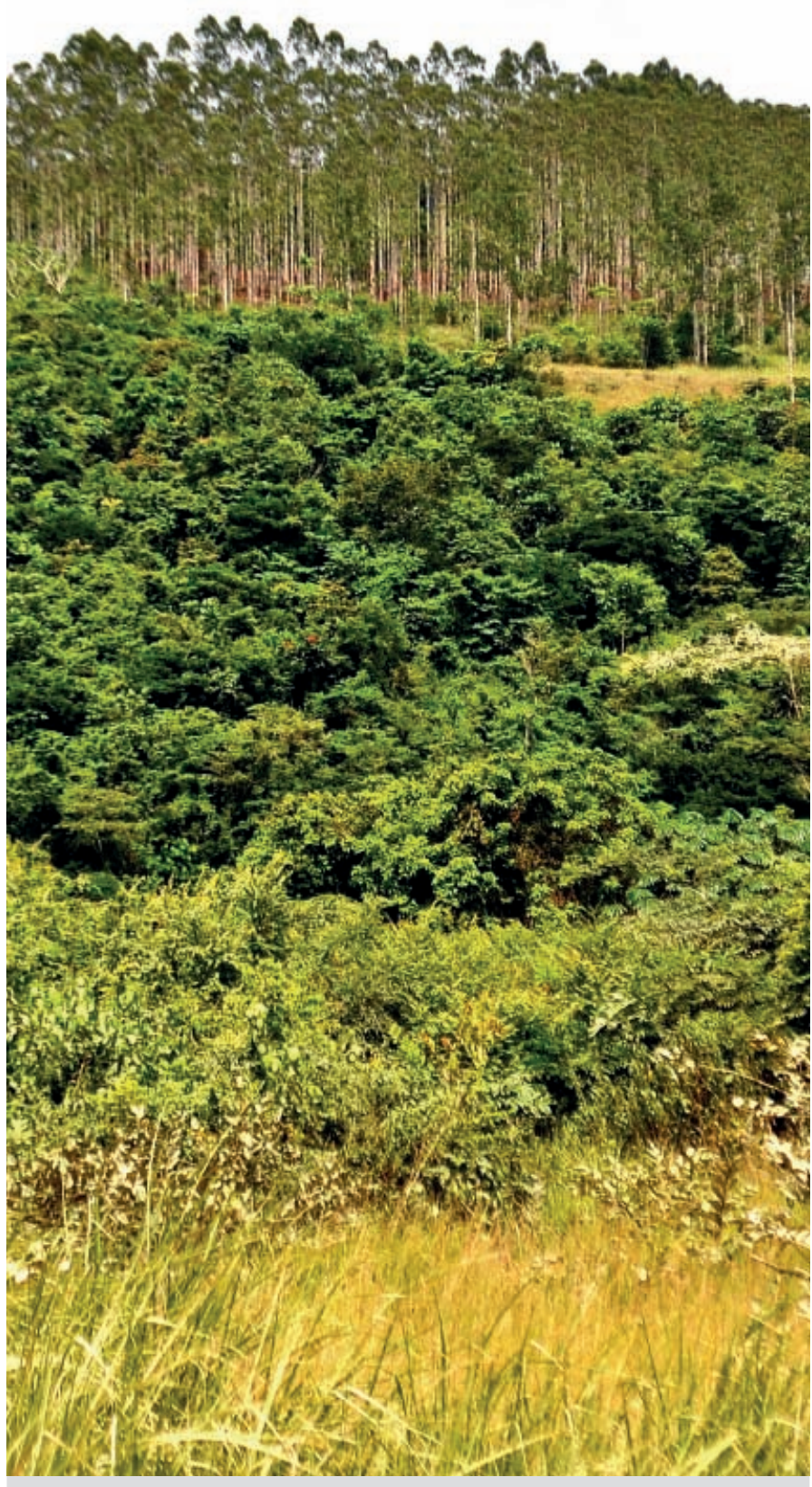

La biodiversité des massifs de plantation d'eucalyptus doit s'envisager à diverses échelles, dont celle du paysage, Pointe-Noire au Congo.

Photo J. Tassin.

\section{Le choix de modèles de référence}

Sur quelles références consensuelles, sinon objectives, est-il possible de se baser pour évaluer la biodiversité d'une plantation d'eucalyptus ? Faut-il se référer aux habitats forestiers naturels les plus proches, ou bien au contraire aux milieux les plus anthropisés ? Un tel choix oscille entre les visions conservatrice et utilitariste de la nature, et demeure politique. Il est toutefois possible d'avancer quelques éléments de réflexion en la matière. 


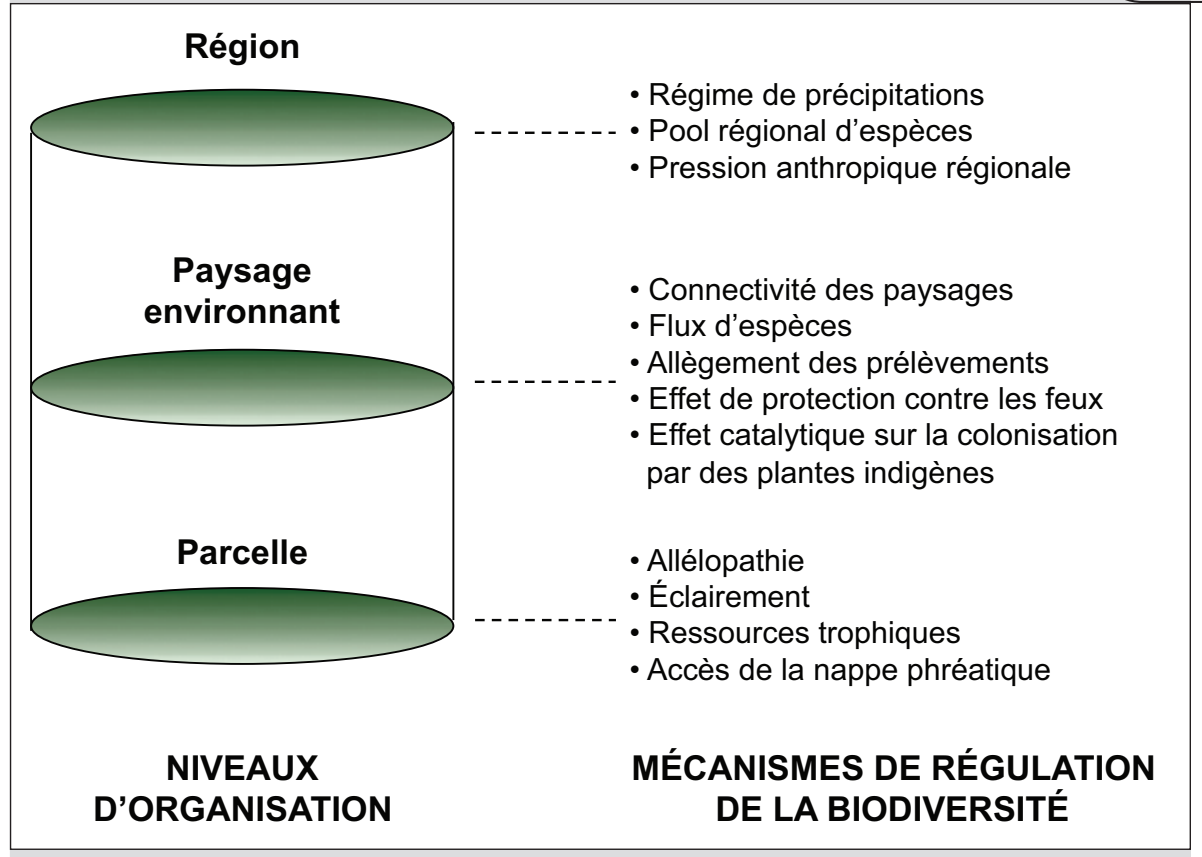

Figure 1.

Mécanismes écologiques régulant la biodiversité sous plantation

d'eucalyptus, positionnés selon les trois principaux niveaux d'organisation.

térêt adaptatif, agronomique ou technologique. À l'inverse, si les plantations sont réalisées sans schéma d'amélioration, il convient de fournir aux planteurs des semis d'espèces pures non améliorées et possédant une variabilité naturelle suffisante pour faire face à d'éventuelles agressions ou stress.

\section{Allélopathie}

L'allélopathie est souvent considérée comme un obstacle majeur à l'expression de la biodiversité sous plantation d'eucalyptus. Feuilles aux arbres et litière au sol y renferment en effet des acides phé-

\section{Processus opérant à l'échelle de la parcelle plantée}

\section{Introduction et maintien de la diversité génétique}

Le genre Eucalyptus est constitué de plus de 600 espèces, dont seulement une vingtaine est utilisée à grande échelle en plantations à travers le monde. La phénoménale diversité de ce genre a permis de bénéficier d'espèces adaptées à des conditions de milieu très diverses. En outre, les hybridations interspécifiques, la sélection d'écotypes et de provenances, mais aussi le clonage d'individus performants et la création de variétés génétiquement modifiées, ont permis de bénéficier de populations optimisées en fonction des stations et des produits attendus (MARIEN, MALLET, 2004). L'essor des marqueurs moléculaires depuis une quinzaine d'années pour analyser la variabilité génétique des essences forestières a contribué à renforcer cette démarche. Ces outils récents ont notamment permis de caractériser les flux de pollen qui opèrent entre individus et populations, la distribution spatiale de la diversité génétique, de même que les effets à long terme des pratiques sylvicoles sur l'évolution de cette diversité génétique (CARnus et al., 2006).

En retour, le niveau de diversité génétique conditionne l'adaptabilité et la résistance de ces populations à des perturbations externes de nature biotique ou abiotique. Une trop grande exiguïté génétique imposée sur de grandes surfaces peut entraîner à long terme des problèmes sanitaires ou des dégâts importants à la suite d'intempéries occasionnelles, mais aussi de changements plus durables des régimes climatiques. Les gestionnaires des plantations d'eucalyptus n'ont alors d'autre alternative que de conserver une diversité naturelle significative pour y puiser les gènes d'in- noliques, des tannins et des flavonoïdes phytotoxiques (Bernhard-ReVERSAT, 1998). Toutefois, ces effets ont été évalués en laboratoire, avec des concentrations élevées qui ne reflètent pas les situations réelles (SUNDER, 1995).

L'abondance du sous-bois dépend avant tout des ressources hydriques, elles-mêmes déterminées au niveau régional, et de leur disponibilité dans le sol prospecté par les racines (SUNDER, 1995). Si la germination et la croissance des plantes de sous-bois sont inhibées sous plantations d'Eucalyptus globulus en Espagne, c'est semble-t-il essentiellement en raison d'un déficit hydrique (Souto et al., 2001).

En outre, l'allélopathie n'est en rien inhérente aux eucalyptus, ni même aux essences exotiques en général. Certains forestiers avancent l'hypothèse que la difficulté à obtenir des peuplements monospécifiques matures d'essences forestières indigènes tient précisément à l'effet d'une telle allélopathie (ZoBEl et al., 1987). C'est notamment le cas des plantations monospécifiques denses de résineux.

\section{Éclairement}

En règle générale, du fait de la silhouette élancée de ces arbres, les plantations d'eucalyptus sont peu fermées. Le manque de lumière n'apparaît donc pas comme un frein direct au développement d'un sous-bois.

En revanche, un excès d'éclairement diffusant au travers de la canopée peut favoriser le développement d'un tapis graminéen qui peut bloquer le développement d'espèces forestières dont les semences sont dispersées à partir de forêts naturelles proches (KANOWSKI et al., 2005). De manière similaire, l'ouverture relative du couvert concourt à l'assèchement aux horizons supérieurs du sol, dont la macrofaune peut en être de ce fait affectée (LOUMETO, BERnHARD-REVERSAT, 1997). 


\section{Processus opérant à l'échelle du paysage environnant}

La structure et la composition du sous-bois constituent des facteurs clés pour l'établissement de la faune, notamment l'avifaune. Au Congo, Brosset (1997) relève une cinquantaine d'espèces d'oiseaux présentes dans des plantations d'eucalyptus disposant d'un sous-bois. Le nombre de ces espèces dans chaque parcelle plantée est positivement corrélé à la richesse spécifique de la flore du sous-bois (BROSSET,1997). L'abondance de la macrofaune du sol est également positivement corrélée au nombre d'espèces de plantes présentes en sous-bois (LoumETo, BERNHARDREVERSAT, 1997). Elle est en outre liée à l'abondance de la litière (LIAO et al., 1984).

Des distinctions doivent être réalisées au sein de l'avifaune, dont les différentes guildes ne partagent pas les mêmes ressources. Les oiseaux fréquentant préférentiellement les espaces herbeux sont souvent exclus des plantations d'eucalyptus (AlLAN et al., 1997). À l'inverse, les passereaux généralistes se contentent d'une parenté de structure entre les plantations d'eucalyptus et les peuplements naturels qu'ils occupent préférentiellement (BROSSET, 1997).

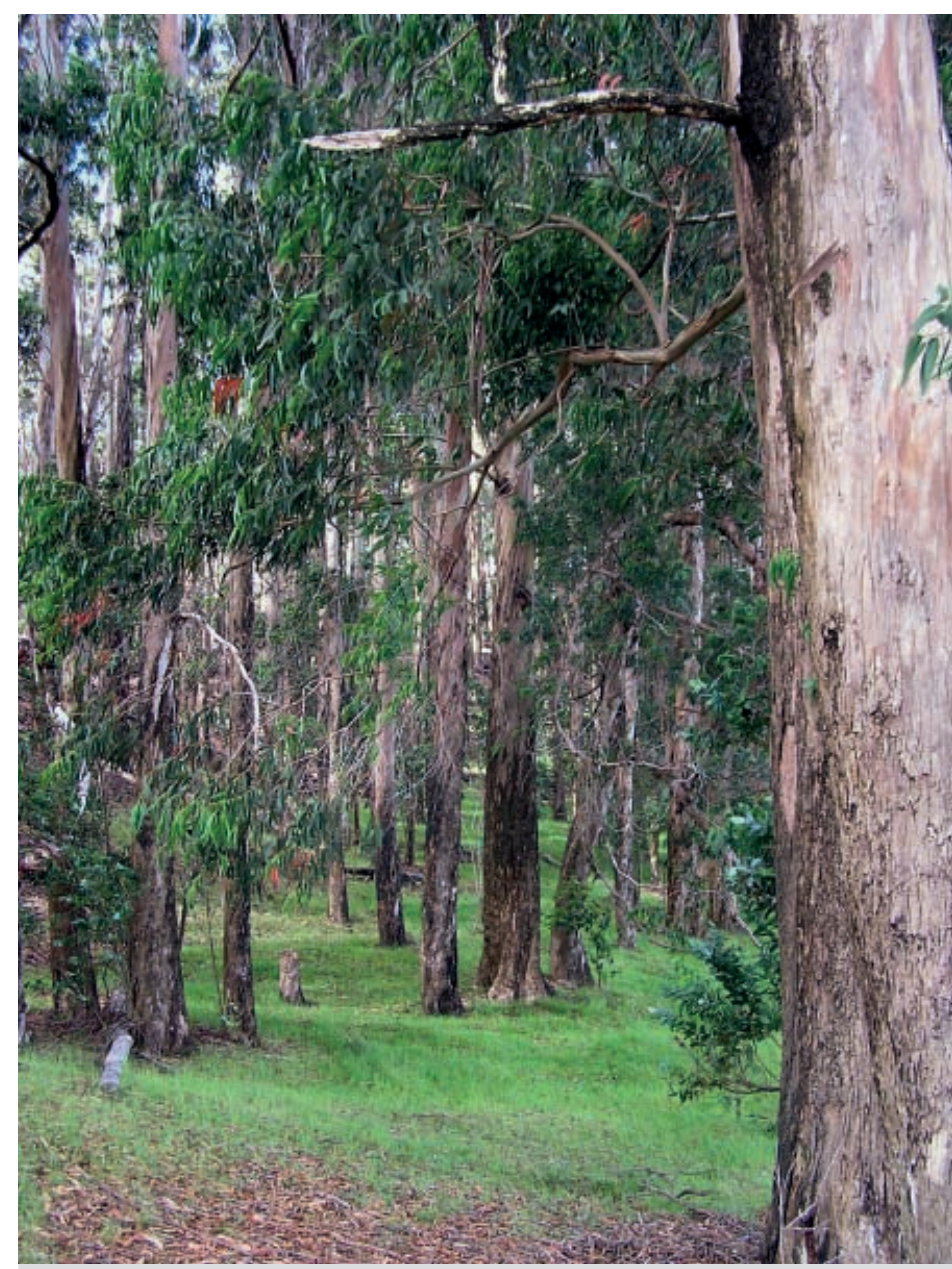

Dans de bonnes conditions climatiques, comme ici à l'île de Pâques, les peuplements d'eucalyptus peuvent être pâturés.

Photo J. Tassin.
Le passage de la parcelle au paysage permet d'intégrer les surfaces plantées et les aires périphériques non plantées, dans la mesure où des interactions s'opèrent entre les deux. La fragmentation des plantations dans le territoire local constitue dès lors un élément très important à prendre en considération dans l'analyse de la diversité. Au Congo, par exemple, les plantations d'eucalyptus, très fragmentées, ne représentent environ que $30 \%$ des territoires concernés, le reste étant constitué de savanes, forêts-galeries, champs, zones humides ou villages. À l'inverse, les grandes plantations industrielles du Brésil couvrent souvent des surfaces d'un seul tenant de plusieurs milliers d'hectares.

\section{Flux d'espèces}

La mise en jeu des agents naturels de dispersion des semences (faune vertébrée, vent, fourmis) permet une colonisation des plantations d'eucalyptus à partir des formations végétales proches, naturelles ou anthropisées (BROSSET, 2001 ; HARRINGTON, EWEL, 1997 ; HutTEL, Loumeto, 2001 ; KANOWSKI et al., 2005). La présence de lambeaux de végétation naturelle au sein ou à proximité du massif sylvicole est déterminante pour la biodiversité patrimoniale observée au sein des plantations d'eucalyptus (GELDENHUYS, 1997 ; YIRDAW, LUUKKANEN, 2003 ; BROCKERHOFF et al., 2008).

A priori, la richesse spécifique des plantations d'eucalyptus n'a jamais été analysée en tenant compte de leur positionnement spatial par rapport aux autres unités paysagères. C'est pourtant un facteur déterminant de la biodiversité observée au sein des parcelles d'eucalyptus. Ainsi, dans les savanes du Niari, au Congo, des buffles, des singes et des antilopes sont régulièrement aperçus dans un peuplement d'eucalyptus de 5000 hectares créé dans les années 1960.

\section{Conversions paysagères}

Le facteur majeur d'érosion de la biodiversité est la disparition des habitats. Un million d'hectares de forêts tropicales sont annuellement convertis en plantations forestières chaque année (FAO, 2005). Il est donc naturel que le reproche majeur visant l'eucalyptus porte en réalité sur les plantations de conversion pour lesquelles des sites naturels sont parfois ainsi transformés, au détriment du patrimoine biologique qu'ils renferment. Cela étant, les plantations d'eucalyptus sont beaucoup plus fréquemment aménagées sur des terrains dégradés ou couverts par des savanes étendues herbeuses, moins riches au plan biologique, mais dont la diversité s'accroît après plantation.

À l'échelle du paysage, les plantations d'essences exotiques peuvent concourir, dans une optique de restauration, à une reconnexion des espaces forestiers et, partant, à faciliter les flux d'espèces forestières. Il faut néanmoins admet- 


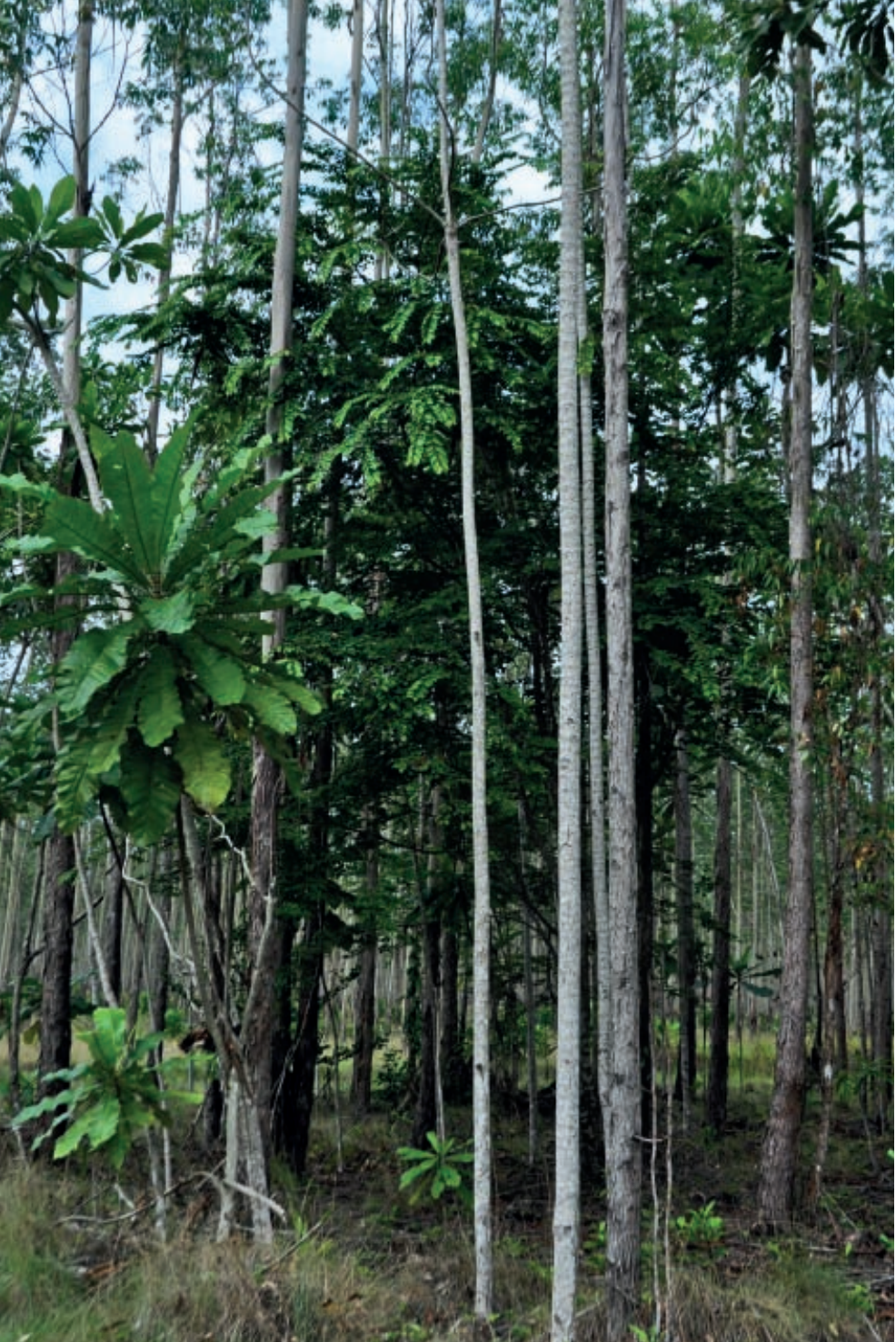

La présence d'espèces indigènes dans le sous-bois dépend de l'éclairement, de l'âge du peuplement, mais aussi de la proximité de sources de semences à l'échelle paysagère, Pointe-Noire au Congo. Photo J. Tassin.

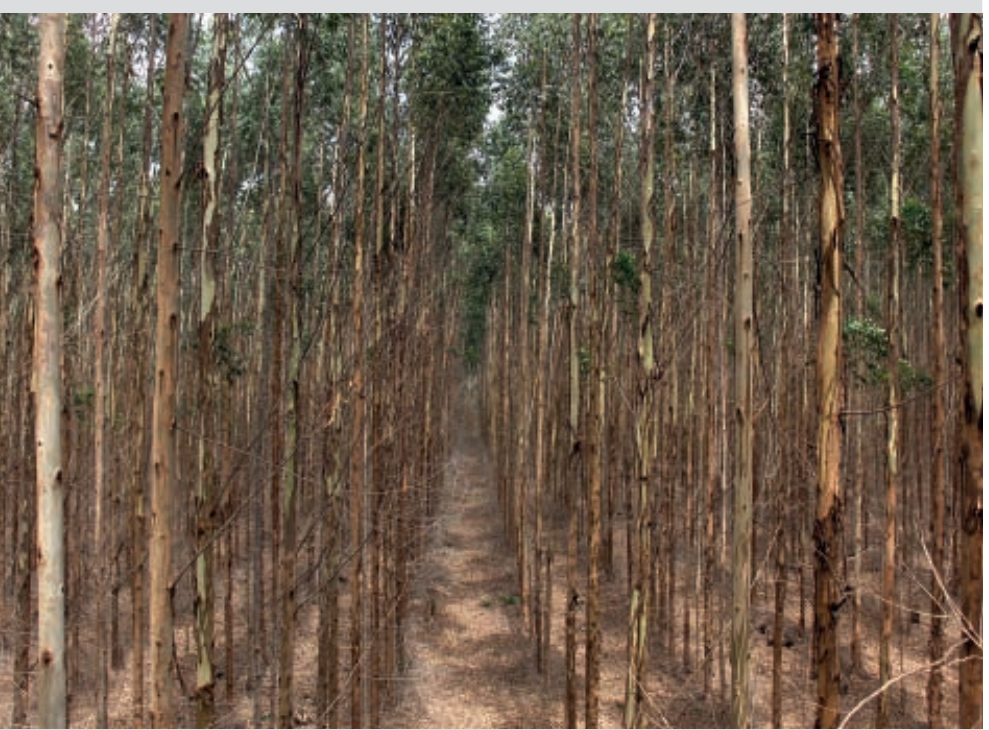

Plantation commerciale d'Eucalyptus grandis au Brésil, Itatinga (État de São Paulo), destinée à la production de panneaux de particules.

Photo J.-P. Laclau. tre que de tels effets dits catalytiques se révèlent faibles sous plantations intensives d'eucalyptus, par comparaison à d'autres essences exotiques (BONE et al., 1997). De manière générale, une telle utilisation de plantations forestières anciennes à des fins de restauration forestière de paysages dégradés reste encore essentiellement expérimentale (GELDENHUYS, 1997).

\section{Effets de protection}

Les plantations d'eucalyptus constituent une ressource ligneuse qui permet d'alléger les pressions sur les forêts naturelles, notamment pour le prélèvement de bois de feu (MAJer, ReCher, 1999). Un tel constat a pu être par exemple réalisé aux abords des capitales, comme AddisAbeba, Antananarivo, Kinshasa et Lomé (SUNDER, 1995).

De même, le contrôle des feux effectué à l'échelle des plantations d'eucalyptus assure un effet de protection à l'égard des habitats naturels voisins, même si elles sont davantage sujettes et perméables au feu que ne le sont les forêts naturelles humides (MAJER, RECHER, 1999).

En outre, des lambeaux de végétation naturelle, difficiles à valoriser à l'échelle industrielle, peuvent être maintenus au sein de massifs plantés d'eucalyptus, contribuant de ce fait à maintenir une mosaïque paysagère favorable à la biodiversité (BRockerHoff et al., 2008). Celle-ci, alors plus élevée à l'échelle du massif de plantation, augmente lorsque la végétation indigène est maintenue dans la zone de plantation (Loyn et al., 2007).

\section{Processus opérant à l'échelle de la région}

\section{Ressources en eau}

Allélopathie locale et ressources en eau régionales ont des effets croisés (ZoBel et al., 1987). Les produits allélochimiques s'accumulent dans le sol mais sont hautement solubles et lessivés par les pluies (MAY, AsH, 1990). Pour des précipitations annuelles supérieures à 1200 millimètres par an, aucun effet négatif n'est observé sur la végétation de sous-bois d'eucalyptus (FAO, 1995).

\section{Pression anthropique régionale}

La proximité d'un centre urbain entre également en considération. La surfréquentation, la divagation d'animaux domestiques n'ayant pas accès à d'autres ressources, la chasse ou le prélèvement de plantes de sous-bois, participent à l'appauvrissement de la diversité biologique sous plantations d'eucalyptus.

Le prélèvement de bois mort peut appauvrir la composition de la litière en invertébrés. Les plantations d'eucalyptus au Sud de la Chine renferment 25 groupes d'invertébrés là où la litière est laissée sur place, et 11 seulement lorsque celleci est au moins partiellement prélevée (LıAo et al., 1984). 


\section{Conclusion et perspectives}

Les plantations d'eucalyptus ne sont pas des forêts naturelles et n'ont pas pour objet d'en être rapprochées. Elles sont vouées à la mise à disposition de produits ligneux, s'agissant de ligniculture, tout comme la viticulture est dévolue à la production du vin, ou la caféiculture à celle du café. Elles correspondent à la satisfaction de besoins pour les populations et l'économie locale ou de marché. La durabilité des plantations d'eucalyptus suppose néanmoins la maîtrise de critères et d'indicateurs de gestion durable écologiques et sociaux, mais aussi économiques et institutionnels.

D'après l'approche hiérarchique, il paraît judicieux que l'ensemble des critères, indicateurs et vérificateurs utilisés en faveur d'une telle gestion durable soient appliqués à tous les niveaux d'agrégation spatiale, de l'arbre à la région. La diversité biologique des plantations d'eucalyptus est en outre directement reliée aux modes de conduite des peuplements et de l'ensemble du massif, pour lesquels des décisions et des recommandations peuvent être édictées à l'échelle locale, mais également nationale ou même internationale. Tout comme les fonctions sociales de ces plantations, il convient de fixer et de concilier les attentes en la matière et d'orienter les techniques, la sylviculture et la gestion dans un sens approprié. Selon une telle perspective, il n'y a pas une seule méthode à mettre en œuvre, mais un ensemble de méthodes, où interviennent notamment la durée de rotation, le nombre de coupes et de rejets viables, le choix des traitements du sol et de la flore accompagnatrice, l'intensité des éclaircies et l'aménagement d'une mosaïque paysagère, qui résultent d'un choix stratégique préalable conciliant les exigences de l'économie, de la société et de l'environnement.

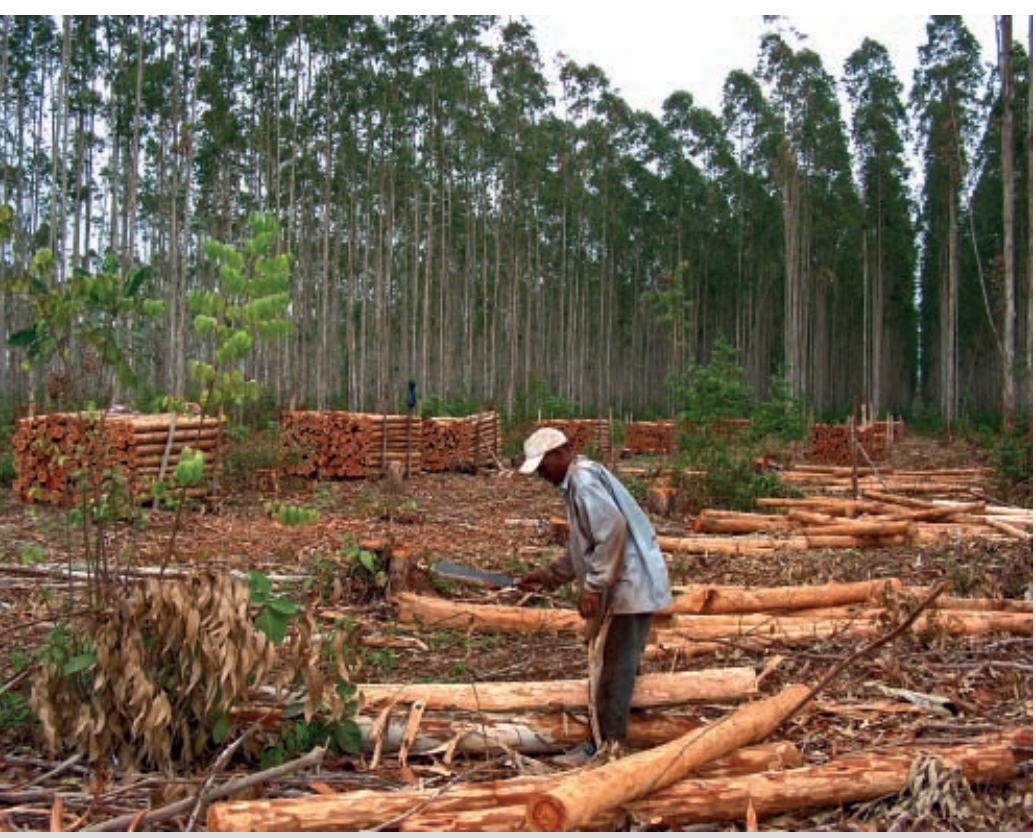

Les plantations d'eucalyptus ne sont pas des forêts naturelles mais correspondent à la satisfaction de besoins, Pointe-Noire au Congo.

Photo D. Louppe.
ALLAN D. J., HARRISON J. A., NAVARRO R. A., VAN WILGEN B. W., THOMPSON M. W., 1997. The impact of commercial afforestation on bird populations in Mpumalanga province, South Africa - insights from bird-atlas data. Biological Conservation, 79: 173-185.

ALLEN T. F. H., STARR T. B., 1982. Hierarchy: perspectives for ecological complexity. Chicago, États-Unis, The University of Chicago Press, $310 \mathrm{p}$.

BERNHARD-REVERSAT F., 1998. Change in $\mathrm{CO}_{2}$ release relationships with initial litter quality during early laboratory decomposition of tropical leaf litters. European Journal of Soil Biology, 34: 117-122.

BERNHARD-REVERSAT F., LOUMETO J. J., LACLAU J.-P., 2001. Litterfall, litter quality and decomposition changes with eucalypt hybrids and plantation age. In : Bernhard-Reversat F. (éd.). Effect of exotic tree plantations on plant diversity and biological soil fertility in the Congo savanna with special reference to eucalypts. Bogor, Indonésie, Center for International Forestry Research, p. 22-29.

BONE R., LAWRENCE M., MAGOMBO Z., 1997. The effect of Eucalyptus camaldulensis (Dehn) plantation on native woodland recovery on Ulumba Mountain, southern Malawi. Forest Ecology and Management, 99: 83-99.

BROCKERHOFF E. G., JACTEL H., PARROTTA J. A., QUINE C. P., SAYER J., 2008. Plantation forests and biodiversity: oxymoron or opportunity? Biodiversity and Conservation, 17: 925-951.

BROSSET A., 2001. Effect of exotic tree plantations on vertebrate fauna. In : Bernhard-Reversat F. (éd.). Effect of exotic tree plantations on plant diversity and biological soil fertility in the Congo savanna: with special reference to eucalypts. Jakarta, Indonésie, Center for International Forestry Research, p. 19-21.

BROSSET A., 1997. Peuplement en oiseaux des plantations d'eucalyptus dans la région de Pointe-Noire, Congo. La Terre et la Vie (Revue d'écologie), 52 (2) : 133-151.

CARNUS J.-M., PARROTTA J., BROCKERHOFF E., ARBEZ M., JACTEL H., KREMER A., LAMBA D., O'HARA K., WALTERS B., 2006. Planted forests and biodiversity. Journal of Forestry, 104 (2): 65-77.

EVANS J., 1992. Plantation forestry in the tropics. $2^{\text {nd }}$ ed. Oxford, Royaume-Uni, Clarendon Press, $432 \mathrm{p}$.

FAO, 1995. Proceedings of the Regional expert consultation on Eucalyptus, 4-8 octobre 1993. Volume I. Bangkok, Thaïlande, FAO Regional Office for Asia and the Pacific, 196 p.

FAO, 2005. Global Forest Resources Assessment 2005. Rome, Italie, Fao, www.fao.org/forestry.

GELDENHUYS C. J., 1997. Native forest regeneration in pine and eucalypt plantations in Northern Province, South Africa. Forest Ecology and Management, 99: 101-115. 
HARRINGTON R. A., EWEL J. J., 1997. Invasibility of tree plantations by native and non-indigenous plant species in Hawaii. Forest Ecology and Management, 99: 153-162.

HUTTEL C., LOUMETO J. L., 2001. Effect of exotic tree plantations and site management on plant diversity. In : BernhardReversat F. (éd.). Effect of exotic tree plantations on plant diversity and biological soil fertility in the Congo savanna: with special reference to eucalypts. Jakarta, Indonésie, Center for International Forestry Research, p. 9-18.

KANOWSKI J., CATTERALL C. P., WARDELL-JOHNSON G. W., 2005. Consequences of broadscale timber plantations for biodiversity in cleared rainforest landscapes of tropical and subtropical Australia. Forest Ecology and Management, 208: 359-372.

LIAO C. H., CHEN M. Q., XIE Y. S., 1984. The initial investigation on soils animals in the tropical artificial forest of Xialiang. Tropical and Subtropical Forestrv Ecosystems, 2: 214-226.

LOUMETO J. J., BERNHARD-REVERSAT F., 1997. La biodiversité dans les plantations d'arbres à croissance rapide au Congo. Bois et Forêts des Tropiques, 253 : 57-61.

LOUMETO J. J., HUTTEL C., 1997. Understory vegetation in fast-growing tree plantations on savanna soils in Congo. Forest Ecology and Management, 99 (1-2): 65-81.

LOYN R. H., MCNABB E. G., MACAK P., NOBLE P., 2007. Eucalypt plantations as habitat for birds on previously cleared farmland in southeastern Australia. Biological Conservation, 137: 533-548.

MAJER J. D., RECHER H. F., 1999. Are eucalypts Brazil's friend or foe? An entomological viewpoint. Anais da Sociedade Entomologica do Brasil, 28 (2): 185-200.

MARIEN J.-N., 2000. Critères et indicateurs de gestion durable pour les plantations d'eucalyptus. L'exemple sud-européen. Bois et Forêts des Tropiques, 266 (4) : 35-42.

MARIEN J.-N., MALLET B., 2004. Nouvelles perspectives pour les plantations forestières en Afrique centrale. Bois et Forêts des Tropiques, 282 : 67-79.

MAY F. E., ASH J. E., 1990. An assessment of the allelopatic potential of Eucalyptus. Australian Journal of Botany, 38: 245-254.

NATIONS UNIES, 1992. Convention on biological diversity, 17 juillet 1992. New York, États-Unis, Nations unies. www.biodiv.org

NOSS R. F., 1990. Indicators for monitoring biodiversity: a hierarchical approach. Conservation Biology, 4 (4): 355-364.

PICKETT S. T. A., KOLASA J., ARMESTO J. J., COLLINS S. L., 1989. The ecological concept of disturbance and its expression at various hiearchical levels. Oikos, 54: 129-136.

POORE M. E. D., FRIES C., 1985. Les effets écologiques des eucalyptus. Rome, Italie, Fao, Étude Forêts nº 59, 118 p.
SOUTO X., BOLANO J. C., GONZALLEZ L., REIGOSA M. J., 2001. Allelophatic effects of tree species on some soil microbial populations and herbaceous plants. Biologia Plantarium, 44: 269-275.

SUNDER S. S., 1995. The ecological, economic and social effects of Eucalyptus. In : White K., Ball J., Kashio M. (éds). Proceedings of the Regional expert consultation on Eucalyptus, 4-8 octobre 1993. Bangkok, Thaïlande, FAO Regional Office for Asia and the Pacific.

WARREN C. R., 2007. Perspectives on the "alien" versus "native" species debate: a critique of concepts, language and practice. Progress in Human Geography, 31 (4): 427-446.

YIRDAW E., LUUKKANEN O., 2003. Indigenous woody species diversity in Eucalyptus globulus Labill. spp. globulus plantations in the Ethiopian highlands. Biodiversity and Conservation, 12: 567-582.

ZOBEL B. J., VAN WYK G., STAHL P., 1987. Growing exotic species. New York, États-Unis, John Wiley \& Sons, 508 p. 